TRANSACTIONS OF THE

AMERICAN MATHEMATICAL SOCIETY

Volume 351, Number 6, Pages 2459-2478

S 0002-9947(99)01985-6

Article electronically published on January 27, 1999

\title{
HARDY INEQUALITIES IN ORLICZ SPACES
}

\author{
ANDREA CIANCHI
}

\begin{abstract}
We establish a sharp extension, in the framework of Orlicz spaces, of the ( $n$-dimensional) Hardy inequality, involving functions defined on a domain $G$, their gradients and the distance function from the boundary of $G$.
\end{abstract}

\section{IntRoduction AND MAIN RESUlTS}

The standard $n$-dimensional Hardy inequality states that, if $G$ is an open bounded subset of euclidean space $\mathbb{R}^{n}$ having a smooth boundary $\partial G$ and $d(x)$ denotes the distance of the point $x \in G$ from $\partial G$, then for every $p \in[1, \infty]$ and $\alpha>-1+1 / p$ a positive constant $C$ exists such that

$$
\left\|\frac{u}{d^{1+\alpha}}\right\|_{L^{p}(G)} \leq C\left\|\frac{D u}{d^{\alpha}}\right\|_{L^{p}(G)}
$$

for all sufficiently smooth functions $u$ vanishing on $\partial G$. Here $L^{p}(G)$ denotes Lebesgue space and $D$ stands for gradient.

A counterpart of (1.1) in the case where functions $u$, which do not necessarily vanish on $\partial G$, are taken into account is the inequality

$$
\left\|\frac{u}{d^{1+\alpha}}\right\|_{L^{p}(G)} \leq C\left(\left\|\frac{u}{d^{\alpha}}\right\|_{L^{p}(G)}+\left\|\frac{D u}{d^{\alpha}}\right\|_{L^{p}(G)}\right),
$$

which holds for $p \in[1, \infty]$ and $\alpha<-1+1 / p$.

A proof of inequalities (1.1)-(1.2) can be found e.g. in [K], [OK]. Let us mention that such inequalities, and generalizations of them, have applications, for example, in the theory of degenerate partial differential equations.

The aim of this present paper is to establish sharp extensions of inequalities (1.1)-(1.2) in the framework of Orlicz spaces. As a consequence of our results, for instance, substitutes for (1.1)-(1.2) in the limiting case where $\alpha=-1+1 / p$ can be derived; see the example below.

Precisely, we are concerned with the problem of finding a necessary and sufficient condition on the real number $\alpha$ and on the Young functions $A$ and $B$ for the inequality

$$
\left\|\frac{u}{d^{1+\alpha}}\right\|_{L^{B}(G)} \leq C\left\|\frac{D u}{d^{\alpha}}\right\|_{L^{A}(G)}
$$

Received by the editors May 15, 1996 and, in revised form, November 15, 1996.

1991 Mathematics Subject Classification. Primary 46E35; Secondary 46 E30.

(C)1999 American Mathematical Society 
to hold for every smooth and bounded open subset $G$ of $\mathbb{R}^{n}$ and all functions $u \in V_{0}^{1, A}\left(G \mid d^{-\alpha}\right)$. Here, $L^{A}(G)$ and $L^{B}(G)$ denote the Orlicz spaces associated with $A$ and $B$, respectively, (definitions are recalled in Section 2) and

$$
\begin{aligned}
& V_{0}^{1, A}\left(G \mid d^{-\alpha}\right)=\{u: u \text { is a real-valued function on } G \text { such that the } \\
& \text { continuation of } u \text { by } 0 \text { outside } G \text { is a weakly differentiable function } \\
& \text { on } \left.\mathbb{R}^{n} \text { and }|D u| d^{-\alpha} \in L^{A}(G)\right\} .
\end{aligned}
$$

An analogous question is considered concerning the inequality

$$
\left\|\frac{u}{d^{1+\alpha}}\right\|_{L^{B}(G)} \leq C\left(\left\|\frac{u}{d^{\alpha}}\right\|_{L^{A}(G)}+\left\|\frac{D u}{d^{\alpha}}\right\|_{L^{A}(G)}\right)
$$

for $u \in W^{1, A}\left(G \mid d^{-\alpha}\right)$, where

$$
\begin{aligned}
& W^{1, A}\left(G \mid d^{-\alpha}\right)=\{u: u \text { is a real-valued weakly differentiable } \\
& \text { function on } \left.G \text { such that } u d^{-\alpha} \text { and }|D u| d^{-\alpha} \in L^{A}(G)\right\} .
\end{aligned}
$$

Answers to these problems are given by Theorems 1 and 2 below. In the statements, $C^{0,1}$ denotes the class of all open bounded subsets of $R^{n}, n \geq 1$, having a Lipschitz-continuous boundary (a precise definition is given in Section 3). Moreover, $\tilde{A}$ denotes the Young conjugate of the function $A$. Throughout the paper, we use the conventions that $1 / \infty=0,1 / 0=\infty$ and $0 \cdot \infty=0$, as usual.

Theorem 1. Let $A$ and $B$ be Young functions and let $\alpha>-1$. Then for every $G \in C^{0,1}$ there exists a positive constant $C$ such that

$$
\left\|\frac{u}{d^{1+\alpha}}\right\|_{L^{B}(G)} \leq C\left\|\frac{D u}{d^{\alpha}}\right\|_{L^{A}(G)}
$$

for all $u \in V_{0}^{1, A}\left(G \mid d^{-\alpha}\right)$ if and only if either

$$
\begin{gathered}
\alpha>0 \text { and there exist numbers } k>0 \text { and } \bar{s} \geq 0 \text { such that } \\
\qquad B(s) \leq A(k s) \text { for } s \geq \bar{s},
\end{gathered}
$$

or

$$
\begin{aligned}
& \alpha=0 \text { and there exist numbers } k>0 \text { and } \bar{s} \geq 0 \text { such that } \\
& \qquad s \int_{\bar{s}}^{s} \frac{B(r)}{r^{2}} d r \leq A(k s) \quad \text { for } s \geq \bar{s}
\end{aligned}
$$

or

$$
\begin{gathered}
-1<\alpha<0 \text { and there exist numbers } k>0 \text { and } \bar{s} \geq 0 \text { such that } \\
\left(\int_{k s}^{\infty}\left(\frac{r}{A(r)}\right)^{-1-1 / \alpha} d r\right)^{-\alpha}\left(\int_{\bar{s}}^{s} \frac{B(r)}{r^{1+1 /(\alpha+1)}} d r\right)^{\alpha+1} \leq k \quad \text { for } s \geq \bar{s}
\end{gathered}
$$

If $\alpha \leq-1$, inequality (1.5) cannot be true whatever $A$ and $B$ are.

Theorem 2. Let $A$ and $B$ be Young functions and let $\alpha<0$. Then for every $G \in C^{0,1}$ there exists a positive constant $C$ such that

$$
\left\|\frac{u}{d^{1+\alpha}}\right\|_{L^{B}(G)} \leq C\left(\left\|\frac{u}{d^{\alpha}}\right\|_{L^{A}(G)}+\left\|\frac{D u}{d^{\alpha}}\right\|_{L^{A}(G)}\right)
$$


for all $u \in W^{1, A}\left(G \mid d^{-\alpha}\right)$ if and only if either

$\alpha<-1$ and there exist numbers $k>0$ and $\bar{s} \geq 0$ such that

$$
B(s) \leq A(k s) \quad \text { for } s \geq \bar{s},
$$

or

$$
\begin{gathered}
\alpha=-1 \text { and there exist numbers } k>0 \text { and } \bar{s} \geq 0 \text { such that } \\
\qquad s \int_{\bar{s}}^{s} \frac{\tilde{A}(r)}{r^{2}} d r \leq \tilde{B}(k s) \quad \text { for } s \geq \bar{s},
\end{gathered}
$$

or

$$
\begin{gathered}
-1<\alpha<0 \text { and there exist numbers } k>0 \text { and } \bar{s} \geq 0 \text { such that } \\
\left(\int_{k s}^{\infty}\left(\frac{r}{\tilde{B}(r)}\right)^{-1+1 /(\alpha+1)} d r\right)^{\alpha+1}\left(\int_{\bar{s}}^{s} \frac{\tilde{A}(r)}{r^{1-1 / \alpha}} d r\right)^{-\alpha} \leq k \quad \text { for } s \geq \bar{s}
\end{gathered}
$$

As a consequence of Theorems 1 and 2, the couples of real numbers $\alpha$ and Young functions $A$ having the property that inequality (1.5) or (1.9) holds with $A=B$ can be characterized. The notion of $\Delta_{2}$-condition for Young functions appearing in the statements of Corollaries 1 and 2 is recalled in the next section.

Corollary 1. Let $A$ be a Young function and let $\alpha>-1$. Then for every $G \in C^{0,1}$ there exists a positive constant $C$ such that

$$
\left\|\frac{u}{d^{1+\alpha}}\right\|_{L^{A}(G)} \leq C\left\|\frac{D u}{d^{\alpha}}\right\|_{L^{A}(G)}
$$

for all $u \in V_{0}^{1, A}\left(G \mid d^{-\alpha}\right)$ if and only if the (generalised right-continuous) inverse $A^{-1}$ of $A$ satisfies

$$
\lim _{\lambda \rightarrow+\infty} \frac{\log \left(\lim \sup _{r \rightarrow+\infty} \frac{A^{-1}(\lambda r)}{A^{-1}(r)}\right)}{\log \lambda}<1+\alpha .
$$

In particular, (1.13) holds for every $A$ in case $\alpha>0$ and holds if and only if $\tilde{A}$ satisfies the $\Delta_{2}$-condition near infinity in case $\alpha=0$. Inequality (1.13) cannot be true if $\alpha \leq-1$ whatever $A$ is.

Corollary 2. Let $A$ be a Young function and let $\alpha<0$. Then for every $G \in C^{0,1}$ there exists a positive constant $C$ such that

$$
\left\|\frac{u}{d^{1+\alpha}}\right\|_{L^{A}(G)} \leq C\left(\left\|\frac{u}{d^{\alpha}}\right\|_{L^{A}(G)}+\left\|\frac{D u}{d^{\alpha}}\right\|_{L^{A}(G)}\right)
$$

for all $u \in W^{1, A}\left(G \mid d^{-\alpha}\right)$ if and only if

$$
\lim _{\lambda \rightarrow+\infty} \frac{\log \left(\liminf _{r \rightarrow+\infty} \frac{A^{-1}(\lambda r)}{A^{-1}(r)}\right)}{\log \lambda}>1+\alpha .
$$

In particular, (1.15) holds for every $A$ in case $\alpha<-1$ and holds if and only if $A$ satisfies the $\Delta_{2}$-condition near infinity in case $\alpha=-1$.

Let us notice that the quantities on the left-hand sides of (1.14) and (1.16) agree with the upper and the lower Boyd index, respectively, of $L^{A}(G)$ (see $[\mathrm{B}]$ ). Such indices are involved in the theory of interpolation. 
Example: limiting cases of inequalities (1.1)-(1.2). Let $G \in C^{0,1}$. Consider inequality (1.5) with $L^{B}(G)=L^{p}(G)$. Then Theorem 1 and Corollary 1 tell us the following.

If $\alpha>0$, then (1.5) holds with $L^{A}(G)=L^{p}(G)$ for every $p \in[1, \infty]$.

When $\alpha=0$, inequality (1.5) holds with $L^{A}(G)=L^{p}(G)$ if $p \in(1, \infty]$ and with $A(s)=s \log (e+s)$ if $p=1$.

When $-1<\alpha<0$, (1.5) holds with $L^{A}(G)=L^{p}(G)$ if $p \in(1 /(\alpha+1), \infty]$, with $A(s)=(s \log (e+s))^{1 /(\alpha+1)}$ if $p=1 /(\alpha+1)$ and for any $A$ such that $\int^{\infty}(r / A(r))^{-1-1 / \alpha} d r<\infty$ if $p \in[1,1 /(\alpha+1))$.

Consider now inequality (1.9) with $L^{A}(G)=L^{p}(G)$. From Theorem 2 and Corollary 2 we get the following conclusions.

If $\alpha<-1$, then (1.9) holds with $L^{B}(G)=L^{p}(G)$ for every $p \in[1, \infty]$.

When $\alpha=-1,(1.9)$ holds with $L^{B}(G)=L^{p}(G)$ for $p \in[1, \infty)$ and with $B(s)=$ $e^{s}-1$ if $L^{A}(G)=L^{\infty}(G)$.

If $-1<\alpha<0$, (1.9) is true with $L^{B}(G)=L^{p}(G)$ for $p \in[1,1 /(\alpha+1)$ ), with $B(s)=(s / \log (e+s))^{1 /(\alpha+1)}$ if $p=1 /(\alpha+1)$ and for any $B$ such that $\int^{\infty}(r / \tilde{B}(r))^{-1+1 /(\alpha+1)} d r<\infty$ if $p \in(1 /(\alpha+1), \infty]$.

Variants of inequalities (1.5) and (1.9) can be considered. In Section 4, we prove a result concerning one-dimensional inequalities which extends to the Orlicz space setting the inequality originally proved by Hardy in $[\mathrm{H}]$ and enables us to prove the necessity of the conditions given in Theorems 1 and 2 . Here, we present substitutes for inequalities (1.5) and (1.9) in the case where the smoothness assumptions on $G$ are relaxed and domains from the class $C^{0, \gamma}$ of all open bounded subsets of $\mathbb{R}^{n}$ whose boundary is Hölder-continuous with exponent $\gamma$ are taken into account.

Theorem 3. Let $A$ and $B$ be Young functions and let $\alpha \in \mathbb{R}$. Assume that $G \in$ $C^{0, \gamma}$ for some $\gamma \in(0,1]$. Set

$$
\sigma= \begin{cases}1 & \text { if either } \alpha \geq 0 \text { or } \alpha \leq-1 \\ \gamma & \text { otherwise. }\end{cases}
$$

(i) If one of the conditions (1.6)-(1.8) holds, then a positive constant $C$ exists such that

$$
\left\|\frac{u}{d^{\gamma(1+\alpha)}}\right\|_{L^{B}(G)} \leq C\left\|\frac{D u}{d^{\alpha \sigma}}\right\|_{L^{A}(G)}
$$

for all $u \in V_{0}^{1, A}\left(G \mid d^{-\alpha \sigma}\right)$.

(ii) If one of the conditions (1.10)-(1.12) holds, then a positive constant $C$ exists such that

$$
\left\|\frac{u}{d^{\sigma(1+\alpha)}}\right\|_{L^{B}(G)} \leq C\left(\left\|\frac{u}{d^{\alpha \gamma}}\right\|_{L^{A}(G)}+\left\|\frac{D u}{d^{\alpha \gamma}}\right\|_{L^{A}(G)}\right)
$$

for all $u \in W^{1, A}\left(G \mid d^{-\alpha \gamma}\right)$.

\section{An interpolation THEOREM}

A basic tool in the proofs of our results is an interpolation theorem which will be established in Subsection 2.2. First, in Subsection 2.1, we recall a few definitions and properties about Young functions, Orlicz spaces and quasilinear operators which will be used in the sequel. 
2.1. A Young function $A$ is a function from $[0, \infty)$ into $[0, \infty]$ having the form

$$
A(s)=\int_{0}^{s} a(r) d r \quad \text { for } s \geq 0
$$

where $a:[0, \infty) \rightarrow[0, \infty]$ is an increasing, left-continuous function which is neither identically zero nor identically infinite on $(0, \infty)$. Notice that

$$
\frac{A(s)}{s} \leq a(s) \leq \frac{A(2 s)}{s} \text { for } s>0 .
$$

The (generalized) right-continuous inverse $A^{-1}$ of $A$ is defined on $[0, \infty]$ as

$$
A^{-1}(r)=\inf \{s: A(s)>r\} \quad(\inf \varnothing=\infty) .
$$

The Young conjugate $\tilde{A}$ of $A$ is the Young function given by

$$
\tilde{A}(s)=\sup \{s r-A(r): r>0\} .
$$

Obviously,

$$
r s \leq A(r)+\tilde{A}(s) \text { for every } r, s \geq 0 .
$$

Moreover, $\tilde{\tilde{A}}=A$.

The following inequalities hold for any Young function $A$

$$
r \leq A^{-1}(r) \tilde{A}^{-1}(r) \leq 2 r \text { for } 0 \leq r \leq \infty .
$$

A Young function $A$ is said to satisfy the $\Delta_{2}$-condition globally [resp. near infinity] if a positive number $c$ exists such that

$$
A^{-1}(r) \geq 2 A^{-1}(c r)
$$

for $r \geq 0$ [resp. for $r$ greater than some positive number].

Let $(M, \nu)$ be a positive measure space (which throughout the paper will be always assumed to be non-atomic and $\sigma$-finite) and let $A$ be a Young function. The Orlicz space $L^{A}(M, \nu)$ is the set of all (equivalence classes) of $\nu$-measurable real-valued functions $f$ on $M$ whose Luxemburg norm $\|f\|_{L^{A}(M, \nu)}$, defined as

$$
\|f\|_{L^{A}(M, \nu)}=\inf \left\{\lambda>0: \int_{M} A\left(\frac{|f(x)|}{\lambda}\right) d \nu \leq 1\right\},
$$

is finite. $L^{A}(M, \nu)$, equipped with the norm $\|\cdot\|_{L^{A}(M, \nu)}$, is a Banach space. Clearly, Lebesgue spaces can be recovered as special cases of Orlicz spaces. Indeed, $L^{A}(M, \nu)=L^{p}(M, \nu)$ if either $p \in[1, \infty)$ and $A(s)=s^{p}$, or $p=\infty$ and $A(s) \equiv 0$ for $0 \leq s \leq 1, A(s) \equiv \infty$ otherwise. In our applications we shall be mainly interested in the case where $M$ is a subset $G$ of $\mathbb{R}^{n}$ and $\nu$ is Lebesgue measure. In this case we shall denote $\nu$ by $m_{n}$ and $L^{A}\left(G, m_{n}\right)$ simply by $L^{A}(G)$.

The following inequality is a substitute for Hölder's inequality in the framework of Orlicz spaces

$$
\int_{M} f(x) g(x) d \nu \leq 2\|f\|_{L^{A}(M, \nu)}\|g\|_{L^{\tilde{A}}(M, \nu)} .
$$

On the other hand, we have

$$
\|f\|_{L^{A}(M, \nu)} \leq \sup \left\{\int_{M} f(x) g(x) d \nu /\|g\|_{L^{\tilde{A}}(M, \nu)}: g \in L^{\tilde{A}}(M, \nu)\right\} .
$$

Let $A$ and $B$ be Young functions. A positive constant $C$ exists such that

$$
\|f\|_{L^{A}(M, \nu)} \leq C\|f\|_{L^{B}(M, \nu)}
$$


for all $f \in\|f\|_{L^{B}(M, \nu)}$ if and only if either $B$ dominates $A$ globally or $\nu(M)<\infty$ and $B$ dominates $A$ near infinity. Recall that a function $B$ is said to dominate a function $A$ globally [near infinity] if a positive constant $c$ exists such that

$$
A(s) \leq B(c s)
$$

for $s \geq 0$ [for $s$ greater than some positive number]. The functions $A$ and $B$ are called equivalent globally [near infinity] if each dominates the other globally [near infinity]. If for every $c>0$ a number $s_{c} \geq 0$ exists such that inequality (2.10) holds for $s \geq s_{c}$, then $A$ is said to increase essentially more slowly than $B$.

For more details and proofs of results about Young functions and Orlicz spaces we refer to $[\mathrm{A}],[\mathrm{BS}]$ and $[\mathrm{RR}]$.

Let $\left(M_{1}, \nu_{1}\right)$ and $\left(M_{2}, \nu_{2}\right)$ be positive measure spaces and let $T$ be an operator whose domain is some linear subspace of the set of $\nu_{1}$-measurable functions on $M_{1}$ and whose range is contained in the set of $\nu_{2}$-measurable functions on $M_{2}$. Then $T$ is called quasilinear if a constant $c \geq 1$ exists such that

$$
|T(f+g)(x)| \leq c(|T f(x)|+|T g(x)|) \quad \text { and } \quad|T(\lambda f)(x)|=|\lambda||T f(x)|
$$

for $\nu_{1}$-a.e. $x \in M_{1}$, for all $f$ and $g$ in the domain of $T$ and all $\lambda \in \mathbb{R}$.

Let $1 \leq p \leq \infty$ and let $T$ be a quasilinear operator as above whose domain contains $L^{p}\left(M_{1}, \nu_{1}\right)$. Then $T$ is said to be of (strong) type $(p, p)$ if a positive constant $N$ exists such that

$$
\|T f\|_{L^{p}\left(M_{2}, \nu_{2}\right)} \leq N\|f\|_{L^{p}\left(M_{1}, \nu_{1}\right)}
$$

for all $f \in L^{p}\left(M_{1}, \nu_{1}\right)$.

For $p \in[1, \infty)$, let $L^{p, 1}\left(M_{1}, \nu_{1}\right)$ denote the Lorentz space of all real-valued $\nu_{1}$ measurable functions $f$ on $M_{1}$ for which the quantity

$$
\|f\|_{L^{p, 1}\left(M_{1}, \nu_{1}\right)}=\int_{0}^{\infty} \nu_{1}\left(\left\{x \in M_{1}:|f(x)|>\tau\right\}\right)^{1 / p} d \tau
$$

is finite. Then $T$ will be called of weak type $(p, p)$ (in the sense of Calderón) if its domain contains $L^{p, 1}\left(M_{1}, \nu_{1}\right)$ and a positive constant $N$ exists such that

$$
\nu_{2}\left(\left\{y \in M_{2}:|T f(y)|>t\right\}\right) \leq N\left(\frac{1}{t}\|f\|_{L^{p, 1}\left(M_{1}, \nu_{1}\right)}\right)^{p}
$$

for all $t>0$ and all $f \in L^{p, 1}\left(M_{1}, \nu_{1}\right)$. The smallest constant $N$ which makes (2.12) or (2.14) true is called the strong or weak $(p, p)$ norm of $T$, respectively.

2.2. Our interpolation theorem can be stated as follows.

Theorem 4. Let $\left(M_{1}, \nu_{1}\right)$ and $\left(M_{2}, \nu_{2}\right)$ be positive, non-atomic and $\sigma$-finite measure spaces and let $T$ be a quasilinear operator whose domain is some linear subspace of the set of $\nu_{1}$-measurable functions on $M_{1}$ and whose range is contained in the set of $\nu_{2}$-measurable functions on $M_{2}$. Let $1 \leq p<\infty$ and assume that $T$ is of weak type $(p, p)$ and of type $(\infty, \infty)$. Let $A$ and $B$ be Young functions.

Then there exists a positive constant $C$, depending only on the $(\infty, \infty)$ and weak $(p, p)$ norms of $T$ and on the constant $c$ appearing in (2.11), such that

$$
\|T f\|_{L^{B}\left(M_{2}, \nu_{2}\right)} \leq C\|f\|_{L^{A}\left(M_{1}, \nu_{1}\right)}
$$

for all $f \in L^{A}\left(M_{1}, \nu_{1}\right)$ if either

(i) $p=1$ and there exists a constant $k>0$ such that

$$
s \int_{0}^{s} \frac{B(r)}{r^{2}} d r \leq A(k s) \quad \text { for } s \geq 0,
$$


or

(ii) $1<p<\infty$ and there exists a constant $k>0$ such that

$$
\left(\int_{k s}^{\infty}\left(\frac{r}{A(r)}\right)^{p^{\prime}-1} d r\right)^{1 / p^{\prime}}\left(\int_{0}^{s} \frac{B(r)}{r^{1+p}} d r\right)^{1 / p} \leq k \quad \text { for } s \geq 0 .
$$

Here $p^{\prime}=p /(p-1)$, the Hölder conjugate of $p$.

In case $\nu_{2}\left(M_{2}\right)<\infty$ the same conclusion is true (with $C$ depending also on $B$ and $\nu_{2}\left(M_{2}\right)$ in (2.15)) even if (2.16) or (2.17) holds only for s larger than some positive number $\bar{s}$ and with 0 replaced by $\bar{s}$ as a lower limit of integration.

Proof. We shall show that a positive constant $k_{1}$ exists having the property that, if $f$ is any $\nu_{1}$-measurable function on $M_{1}$ such that

$$
\int_{M_{1}} A(2 k|f(x)|) d \nu_{1} \leq 1
$$

then $T f$ is well-defined and

$$
\int_{M_{2}} B\left(\frac{|T f(y)|}{2 c k_{1}}\right) d \nu_{2} \leq 1 .
$$

Hence (2.15) will follow with $C=4 c k k_{1}$.

Let $a$ and $b$ be the increasing (left-continuous) functions such that $A(s)=$ $\int_{0}^{s} a(r) d r$ and $B(s)=\int_{0}^{s} b(r) d r$. We shall assume that $A$ and $B$ are strictly increasing and everywhere finite, the general case requiring only minor modifications. Let $f$ be any function satisfying (2.18). Let us set $f_{t}=\operatorname{sign}(f) \min \{t,|f|\}$ and $f^{t}=f-f_{t}$ for $t>0$. Clearly, $f_{t} \in L^{\infty}\left(M_{1}, \nu_{1}\right)$; moreover, $f^{t} \in L^{p, 1}\left(M_{1}, \nu_{1}\right)$. To verify the latter membership, observe that

$$
\begin{aligned}
\left\|f^{t}\right\|_{L^{p, 1}\left(M_{1}, \nu_{1}\right)} & =\int_{t}^{\infty} \nu_{1}(\{|f|>s\})^{1 / p} d s \\
& \leq\left\|\left(\frac{s}{A(2 k s)}\right)^{1 / p}\right\|_{L^{p^{\prime}(t, \infty)}}\left(\int_{t}^{\infty} \nu_{1}(\{|f|>s\}) \frac{A(2 k s)}{s} d s\right)^{1 / p}
\end{aligned}
$$

by Hölder's inequality. Thanks to (2.2), to the equation

$$
2 k \int_{0}^{\infty} \nu_{1}(\{|f|>s\}) a(2 k s) d s=\int_{M_{1}} A(2 k|f(x)|) d \nu_{1}
$$

and to (2.18), the last integral in (2.20) does not exceed 1. Hence, $\left\|f^{t}\right\|_{L^{p, 1}\left(M_{1}, \nu_{1}\right)}<$ $\infty$, since $\left\|(s / A(2 k s))^{1 / p}\right\|_{L^{p^{\prime}}(t, \infty)}$ agrees either with $t / A(2 k t)$ or with the first factor on the left-hand side of (2.17), according to whether $p=1$ or $1<p<\infty$. In conclusion, we have shown that $f=f_{t}+f^{t}$ with $f_{t} \in L^{\infty}\left(M_{1}, \nu_{1}\right)$ and $f^{t} \in$ $L^{p, 1}\left(M_{1}, \nu_{1}\right)$; consequently, $f$ belongs to the domain of $T$.

Now, if $k_{1}$ is any positive number, then (2.11) ensures that

$$
\begin{aligned}
\int_{M_{2}} B\left(\frac{|T f(y)|}{2 c k_{1}}\right) d \nu_{2}= & \int_{0}^{\infty} b(t) \nu_{2}\left(\left\{|T f|>2 c k_{1} t\right\}\right) d t \\
\leq & \int_{0}^{\infty} b(t) \nu_{2}\left(\left\{\left|T f_{t}\right|>k_{1} t\right\}\right) d t \\
& +\int_{0}^{\infty} b(t) \nu_{2}\left(\left\{\left|T f^{t}\right|>k_{1} t\right\}\right) d t
\end{aligned}
$$


Let $N_{\infty}$ denote the $(\infty, \infty)$ norm of $T$. Then

$$
\left\|T f_{t}\right\|_{L^{\infty}\left(M_{1}, \nu_{1}\right)} \leq N_{\infty}\left\|f_{t}\right\|_{L^{\infty}\left(M_{1}, \nu_{1}\right)} \leq N_{\infty} t \text { for } t>0 .
$$

Therefore,

$$
\int_{0}^{\infty} b(t) \nu_{2}\left(\left\{\left|T f_{t}\right|>k_{1} t\right\}\right) d t=0
$$

if $k_{1} \geq N_{\infty}$, since, by $(2.23), \nu_{2}\left(\left\{\left|T f_{t}\right|>k_{1} t\right\}\right) \equiv 0$ for such a choice of $k_{1}$.

On the other hand, denoting by $N_{p}$ the weak $(p, p)$ norm of $T$, we have

$$
\begin{aligned}
\nu_{2}\left(\left\{\left|T f^{t}\right|>k_{1} t\right\}\right) & \leq \frac{N_{p}}{k_{1}^{p} t^{p}}\left(\left\|f^{t}\right\|_{L^{p, 1}\left(M_{1}, \nu_{1}\right)}\right)^{p} \\
& =\frac{N_{p}}{k_{1}^{p} t^{p}}\left(\int_{t}^{\infty} \nu_{1}(\{|f|>s\})^{1 / p} d s\right)^{p} \quad \text { for } t>0 .
\end{aligned}
$$

Hence,

$$
\int_{0}^{\infty} b(t) \nu_{2}\left(\left\{\left|T f^{t}\right|>k_{1} t\right\}\right) d t \leq \frac{N_{p}}{k_{1}^{p}} \int_{0}^{\infty} \frac{b(t)}{t^{p}}\left(\int_{t}^{\infty} \nu_{1}(\{|f|>s\})^{1 / p} d s\right)^{p} d t .
$$

By making use of inequality (2.2) with $A$ replaced by $B$, it is easily deduced from assumption (2.16) or (2.17) that

$$
s \int_{0}^{s} \frac{b(t)}{t} d t \leq A(2 k s) \quad \text { for } s>0
$$

or

$$
\left(\int_{s}^{\infty}\left(\frac{r}{A(2 k r)}\right)^{p^{\prime}-1} d r\right)^{1 / p^{\prime}}\left(\int_{0}^{s} \frac{b(r)}{r^{p}} d r\right)^{1 / p} \leq 1 \quad \text { for } s>0,
$$

respectively. By the weighted Hardy inequality in $L^{p}(0, \infty)$ (see e.g., Theorem 1 of $[\mathrm{M}]$ ), condition (2.27) for $p=1$ and condition $(2.28)$ for $p \in(1, \infty)$ ensure that the right-hand side of $(2.26)$ does not exceed $c_{p} N_{p} k_{1}^{-p} \int_{0}^{\infty} \nu_{1}(\{|f|>s\}) \frac{A(2 k s)}{s} d s$, where $c_{p}$ equals either 1 or $p p^{1 /\left(p^{\prime}-1\right)}$, according to whether $p=1$ or $p \in(1, \infty)$. Hence, owing to (2.2), (2.21) and (2.18),

$$
\int_{0}^{\infty} b(t) \nu_{2}\left(\left\{\left|T f^{t}\right|>k_{1} t\right\}\right) d t \leq c_{p} N_{p} k_{1}^{-p} .
$$

By (2.22), (2.24) and (2.29), inequality (2.19) holds provided that

$$
k_{1} \geq \max \left\{N_{\infty}, N_{p}^{1 / p} c_{p}^{1 / p}\right\}
$$

and hence, in particular, if $k_{1}=\max \left\{N_{\infty}, e^{2 / e}, N_{p} e^{2 / e}\right\}$.

Finally, the assertion concerning the case where $\nu_{2}\left(M_{2}\right)<\infty$ is a straightforward consequence of the fact that, under such an assumption, replacing $B$ by a Young function which is equivalent to $B$ near infinity but vanishes on $[0, \bar{s})$ results in an equivalent norm on $L^{B}\left(M_{2}, \nu_{2}\right)$. 


\section{Proofs of Results from Section 1}

We begin by recalling the precise definition of the class of domains $C^{0, \gamma}$ (see $[\mathrm{K}])$.

Definition. Let $0<\gamma \leq 1$. A bounded open subset $G$ of $\mathbb{R}^{n}, n \geq 1$, is said to belong to the class $C^{0, \gamma}$ if there exist

i) a finite number $N$ of orthogonal coordinate systems $x_{i}=\left(x_{i}^{\prime}, x_{n i}\right)$, where $x_{i}^{\prime}=\left(x_{1 i}, x_{2 i}, \ldots, x_{(n-1) i}\right), i=1, \ldots, N$

ii) $N$ corresponding functions $\phi_{i}\left(x_{i}^{\prime}\right)$, which are Hölder-continuous with exponent $\gamma$ and whose domains are $(n-1)$-dimensional cubes $Q_{i}$

iii) a number $\beta \in(0,1)$

such that, if we set $V_{i}=\left\{x_{i}: x_{i}^{\prime} \in Q_{i}, \phi\left(x_{i}^{\prime}\right)-\beta<x_{n i}<\phi\left(x_{i}^{\prime}\right)+\beta\right\}, U_{i}=V_{i} \cap G$ and $\Gamma_{i}=V_{i} \cap \partial G$, then

$$
\begin{gathered}
\partial G \subset \bigcup_{i=1}^{N} V_{i}, \quad U_{i}=\left\{x_{i}: x_{i}^{\prime} \in Q_{i}, \phi\left(x_{i}^{\prime}\right)<x_{n i}<\phi\left(x_{i}^{\prime}\right)+\beta\right\} \quad \text { and } \\
\Gamma_{i}=\left\{x_{i}: x_{i}^{\prime} \in Q_{i}, x_{n i}=\phi\left(x_{i}^{\prime}\right)\right\} .
\end{gathered}
$$

In particular, in case $n=1, G \in C^{0, \gamma}$ for every $\gamma \in(0,1]$ if and only if $G$ is the union of a finite number of open intervals.

Now, we prove Theorem 1 and Theorem 3 part i). Corollary 1 follows from Theorem 1, via Lemma 4 below. The proofs of Theorem 2 and of Theorem 3 part ii) make use of a partition of unity and of arguments similar to those used in the proof of Theorem 1; for brevity, we omit them. Corollary 2 follows from Theorem 2 , via a "dual" version of Lemma 4.

Proof of Theorem 1. Let $G \in C^{0,1}$ and assume that one of the conditions (1.6)-(1.8) is fulfilled. Let $x_{i}=\left(x_{i}^{\prime}, x_{n i}\right), Q_{i}, \phi_{i}, U_{i}, i=1, \ldots, N$, be as in the definition above with $\gamma=1$. We suppose, for the moment, that $i$ is fixed and denote $x_{i}=\left(x_{i}^{\prime}, x_{n i}\right)$, $Q_{i}, \phi_{i}, U_{i}$ simply by $x=\left(x^{\prime}, x_{n}\right), Q, \phi, U$.

Our first step will be to show that a positive constant $c_{1}$ exists such that

$$
\left\|\left(x_{n}-\phi\left(x^{\prime}\right)\right)^{-1-\alpha} \int_{\phi\left(x^{\prime}\right)}^{x_{n}} v\left(x^{\prime}, \tau\right) d \tau\right\|_{L^{B}(U)} \leq c_{1}\left\|\left(x_{n}-\phi\left(x^{\prime}\right)\right)^{-\alpha} v(x)\right\|_{L^{A}(U)}
$$

for all real-valued functions $v$ on $U$ such that $\left(x_{n}-\phi\left(x^{\prime}\right)\right)^{-\alpha} v(x) \in L^{A}(U)$. Let us call $T_{\alpha}$ the linear operator defined on $w: U \rightarrow \mathbb{R}$ by

$$
T_{\alpha} w(x)=\left(x_{n}-\phi\left(x^{\prime}\right)\right)^{-1-\alpha} \int_{\phi\left(x^{\prime}\right)}^{x_{n}} w\left(x^{\prime}, \tau\right)\left(\tau-\phi\left(x^{\prime}\right)\right)^{\alpha} d \tau \quad \text { for } x \in U .
$$

Clearly, inequality (3.1) is equivalent to

$$
\left\|T_{\alpha} w\right\|_{L^{B}(U)} \leq c_{1}\|w\|_{L^{A}(U)}
$$

for all $w \in L^{A}(U)$.

Since $\alpha>-1$, equation (3.2) immediately implies that

$$
\left\|T_{a} w\right\|_{L^{\infty}(U)} \leq \frac{1}{1+\alpha}\|w\|_{L^{\infty}(U)} .
$$

Thus, $T_{\alpha}$ is of type $(\infty, \infty)$ with norm $\leq 1 /(1+\alpha)$. 
Now, let us distinguish the cases where $\alpha>0$ and $-1<\alpha \leq 0$. If $\alpha>0$, then, via Fubini's theorem, it is easily seen that

$$
\int_{\phi\left(x^{\prime}\right)}^{\phi\left(x^{\prime}\right)+\beta}\left|T_{\alpha} w\left(x^{\prime}, x_{n}\right)\right| d x_{n} \leq \frac{1}{\alpha} \int_{\phi\left(x^{\prime}\right)}^{\phi\left(x^{\prime}\right)+\beta}\left|w\left(x^{\prime}, \tau\right)\right| d \tau \quad \text { for } x^{\prime} \in Q .
$$

On integrating both sides of (3.5) with respect to $x^{\prime}$ over $Q$ we get

$$
\int_{U}\left|T_{\alpha} w(x)\right| d x \leq \frac{1}{\alpha} \int_{U}|w(x)| d x
$$

Thus, $T_{\alpha}$ is of strong type $(1,1)$ with norm $\leq 1 / \alpha$. Since $T_{\alpha}$ is simultaneously of strong type $(1,1)$ and $(\infty, \infty)$, an interpolation theorem by Calderón (see e.g., Theorem 2.a.10 of [LT]) ensures that inequality (3.3) holds with $A=B$; in particular, by (2.9), (3.3) holds if (1.6) is satisfied.

If $-1<\alpha \leq 0$, then Hardy-Littlewood's inequality (see Theorem 2.2, Chapter 2 , of $[\mathrm{BS}]$, for instance) implies that

$$
\left|T_{\alpha} w(x)\right| \leq\left(x_{n}-\phi\left(x^{\prime}\right)\right)^{-1-\alpha} \int_{0}^{\infty} w\left(x^{\prime}, \cdot\right)^{*}(s) s^{\alpha} d x \quad \text { for } x \in U,
$$

where

$$
w\left(x^{\prime}, \cdot\right)^{*}(s)=\sup \left\{\theta \geq 0: m_{1}\left(\left\{x_{n} \in\left(\phi\left(x^{\prime}\right), \phi\left(x^{\prime}\right)+\beta\right):\left|w\left(x^{\prime}, x_{n}\right)\right|>\theta\right\}\right)>s\right\},
$$

the decreasing rearrangement of $w\left(x^{\prime}, x_{n}\right)$ with respect to $x_{n}$. Notice that the integral on the right-hand side of (3.7) equals

$$
\frac{1}{1+\alpha} \int_{0}^{\infty} m_{1}\left(\left\{x_{n} \in\left(\phi\left(x^{\prime}\right), \phi\left(x^{\prime}\right)+\beta\right):\left|w\left(x^{\prime}, x_{n}\right)\right|>\theta\right\}\right)^{1+\alpha} d \theta .
$$

Consequently,

$$
\begin{aligned}
& m_{1}\left(\left\{x_{n} \in\left(\phi\left(x^{\prime}\right), \phi\left(x^{\prime}\right)+\beta\right):\left|T_{\alpha} w\left(x^{\prime}, x_{n}\right)\right|>t\right\}\right) \\
\leq & \left(\frac{1}{(1+\alpha) t} \int_{0}^{\infty} m_{1}\left(\left\{x_{n} \in\left(\phi\left(x^{\prime}\right), \phi\left(x^{\prime}\right)+\beta\right):\left|w\left(x^{\prime}, x_{n}\right)\right|>\theta\right\}\right)^{1+\alpha} d \theta\right)^{1 /(1+\alpha)}
\end{aligned}
$$

for $x^{\prime} \in Q$ and $t>0$.

Integrating both sides of (3.8) with respect to $x^{\prime}$ over $Q$ and making use of Minkowski's integral inequality on the right-hand side yields

$$
m_{n}\left(\left\{x \in U:\left|T_{\alpha} w(x)\right|>t\right\}\right) \leq \frac{1}{((1+\alpha) t)^{1 /(1+\alpha)}}\|w\|_{L^{1 /(1+\alpha), 1}(U)}^{1 /(1+\alpha)} \quad \text { for } t>0 .
$$

Thus, $T_{\alpha}$ is of weak type $(1 /(1+\alpha), 1 /(1+\alpha))$ with norm $\leq(1+\alpha)^{-1 /(1+\alpha)}$. Hence, by Theorem 4, inequality (3.3) is true if either (1.7) or (1.8) is fulfilled.

Now, let $u \in V_{0}^{1, A}\left(G \mid d^{-\alpha}\right)$ and, with abuse of notation, let us still call $u$ this function in the local coordinates $x=\left(x^{\prime}, x_{n}\right)$ on $U$. Since the continuation of $u$ by 0 outside $G$ is a weakly differentiable function on $\mathbb{R}^{n}$, then $u\left(x^{\prime}, \cdot\right)$ is absolutely continuous on $\left(\phi\left(x^{\prime}\right), \phi\left(x^{\prime}\right)+\beta\right)$ for a.e. $x^{\prime} \in Q$ and

$$
u(x)=\int_{\phi\left(x^{\prime}\right)}^{x_{n}} \frac{\partial u}{\partial x_{n}}\left(x^{\prime}, \tau\right) d \tau \quad \text { for a.e. } x \in U .
$$

Since $\phi$ is Lipschitz-continuous, Lemma 4.6 of $[\mathrm{K}]$ ensures that a positive constant $\delta$ exists such that

$$
\delta\left(x_{n}-\phi\left(x^{\prime}\right)\right) \leq d(x) \leq\left(x_{n}-\phi\left(x^{\prime}\right)\right) \quad \text { for } x \in U .
$$


From (3.1), (3.10) and (3.11) we obtain

$$
\left\|d(x)^{-1-\alpha} u(x)\right\|_{L^{B}(U)} \leq c_{3}\left\|d(x)^{-\alpha} D u(x)\right\|_{L^{A}(U)} .
$$

Here $c_{3}=c_{1} c_{2} \delta^{-1-\alpha}$, where $c_{2}$ equals 1 if $\alpha \geq 0$ and agrees with $\delta^{\alpha}$ otherwise.

Let us set $\hat{G}=G \backslash \bigcup_{i=1}^{N} U_{i}$ and call $c_{4}$ the largest of the constants $c_{3}$ appearing in (3.12) as $U$ ranges among all $U_{i}, i=1, \ldots, N$. Then

$$
\begin{aligned}
\left\|d^{-1-\alpha} u\right\|_{L^{B}(G)} & \leq \sum_{i=1}^{N}\left\|d^{-1-\alpha} u\right\|_{L^{B}\left(U_{i}\right)}+\left\|d^{-1-\alpha} u\right\|_{L^{B}(\hat{G})} \\
& \leq N c_{4}\left\|d^{-\alpha} D u\right\|_{L^{A}(G)}+\operatorname{dist}(\hat{G}, \partial G)^{-1-\alpha}\|u\|_{L^{B}(\hat{G})} .
\end{aligned}
$$

Since we are assuming that one of the conditions (1.6)-(1.8) is in force, then, by Lemma 1 below, $A$ dominates $B$ near infinity; thus, a constant $c_{5}$, independent of $u$, exists such that $\|u\|_{L^{B}(\hat{G})} \leq c_{5}\|u\|_{L^{A}(\hat{G})}$. On the other hand, by Lemma 3 below there exists a constant $c_{6}$, independent of $u$, such that $\|u\|_{L^{A}(\hat{G})} \leq$ $c_{6}\left\|d^{-\alpha} D u\right\|_{L^{A}(\hat{G})}$. Combining the last two inequalities shows that inequality (1.5) follows from (3.13) with $C=N c_{4}+c_{5} c_{6} \operatorname{dist}(\hat{G}, \partial G)^{-1-\alpha}$.

Conversely, assume that $\alpha, A$ and $B$ are such that (1.5) holds for every $G \in C^{0,1}$. Let us choose the ball $S$, centered at the origin and having radius 1, as domain $G$. Consider radially symmetric functions $u \in V_{0}^{1, A}\left(S \mid d^{-\alpha}\right)$ having the form

$$
u(x)=\int_{|x|}^{1} f(1-r) d r \quad \text { for } x \in S,
$$

for some $f:[0,1] \rightarrow \mathbb{R}$. Since $d(x)=1-|x|$, inequality (1.5) implies that

$$
\left\|r^{-1-\alpha} \int_{0}^{r} f(\tau) d \tau\right\|_{L^{B}\left((0,1),(1-r)^{n-1}\right)} \leq n C_{n} C\left\|r^{-\alpha} f(r)\right\|_{L^{A}\left((0,1),(1-r)^{n-1}\right)}
$$

where $C_{n}=\pi^{n / 2} / \Gamma(1+n / 2)$, the measure of $S$. Here, $L^{A}\left((0,1),(1-r)^{n-1}\right)$ and $L^{B}\left((0,1),(1-r)^{n-1}\right)$ stand for $L^{A}((0,1), \nu)$ and $L^{B}((0,1), \nu)$, respectively, where $\nu$ is the measure whose density with respect to $m_{1}$ is $(1-r)^{n-1}$. From (3.14) one easily infers that a positive constant $c$ exists such that

$$
\left\|r^{-1-\alpha} \int_{0}^{r} f(\tau) d \tau\right\|_{L^{B}(0,1 / 2)} \leq c\left\|r^{-\alpha} f(r)\right\|_{L^{A}(0,1 / 2)}
$$

for all functions $f$ such that $r^{-\alpha} f(r) \in L^{A}(0,1 / 2)$. Thus, in order to conclude our proof we need only to know that, if the 1-dimensional inequality (3.15) holds, then $\alpha>-1$ and one of the conditions (1.6)-(1.8) is true. This is proved in Theorem 5, Section 4 .

Proof of Theorem 3 i), sketched. The proof proceeds along the same lines as in Theorem 1 . The only difference is that, since $\partial G$ is assumed to be only Höldercontinuous with exponent $\gamma$, then the inequalities

$$
\delta\left(x_{n}-\phi\left(x^{\prime}\right)\right)^{1 / \gamma} \leq d(x) \leq\left(x_{n}-\phi\left(x^{\prime}\right)\right) \quad \text { for } x \in U
$$

hold instead of (3.11) (see Lemma 4.6 of $[\mathrm{K}]$ ). Consequently, the exponent $(1+\alpha)$ of $\delta$ in the definition of the constant $c_{3}$ has to be replaced by $\gamma(1+\alpha)$ and the exponent $\alpha$ of $\delta$ in the definition of $c_{2}$ has to be replaced by $\gamma \alpha$.

Lemma 1. Let $A$ and $B$ be Young functions and let $\alpha \in \mathbb{R}$. Assume that one of the conditions (1.6)-(1.8) is fulfilled. Then $A$ dominates $B$ near infinity. 
Proof. The assertion is obvious if (1.6) is in force. Let us assume that either (1.7) or (1.8) is fulfilled. Then, $-1<\alpha \leq 0$. Let $\bar{s}$ be the number appearing in (1.7) or (1.8), according to whether $\alpha=0$ or $-1<\alpha<0$. The following chain holds

$$
\begin{aligned}
B(s / 2) & =\frac{s^{1 /(1+\alpha)} B(s / 2)}{(1+\alpha)\left(2^{1 /(1+\alpha)}-1\right)} \int_{s / 2}^{s} \frac{1}{r^{1+1 /(1+\alpha)}} d r \\
& \leq \frac{s^{1 /(1+\alpha)}}{(1+\alpha)\left(2^{1 /(1+\alpha)}-1\right)} \int_{s / 2}^{s} \frac{B(r)}{r^{1+1 /(1+\alpha)}} d r \\
& \leq \frac{s^{1 /(1+\alpha)}}{(1+\alpha)\left(2^{1 /(1+\alpha)}-1\right)} \int_{\bar{s}}^{s} \frac{B(r)}{r^{1+1 /(1+\alpha)}} d r \\
& \leq s^{1 /(1+\alpha)} \int_{\bar{s}}^{s} \frac{B(r)}{r^{1+1 /(1+\alpha)}} d r \quad \text { for } s \geq 2 \bar{s} .
\end{aligned}
$$

Therefore, if (1.7) is satisfied, then domination of $A$ over $B$ near infinity follows from (3.17) with $\alpha=0$. Suppose now that $-1<\alpha<0$. In the case where $A$ equals $\infty$ for large values of the argument, then $A$ trivially dominates $B$ near infinity. Conversely, assume that $A$ is everywhere finite and let $s_{0} \geq 0$ be such that $A(s)>0$ if $s>s_{0}$. Then a chain of inequalities similar to (3.17) yields

$$
s^{1 /(1+\alpha)}\left(\int_{s}^{\infty}\left(\frac{r}{A(r)}\right)^{-1-1 / \alpha} d r\right)^{\alpha /(1+\alpha)} \leq A(2 s) \text { for } s>s_{0} .
$$

Inequalities (3.17) and (3.18) imply that, under assumption (1.8), $A$ dominates $B$ near infinity.

Lemma 2. Let $n \geq 2$ and let $A$ be a Young function. Let $A_{n}$ be the Young function defined by

$$
A_{n}(s)=\int_{0}^{s} r^{n^{\prime}-1}\left(\Phi_{n}\left(r^{n^{\prime}}\right)\right)^{n^{\prime}} d r
$$

where $\Phi_{n}(s)=\int_{0}^{s} \tilde{A}(t) t^{-1-n^{\prime}} d t$ and $A$ is replaced, if necessary, by an equivalent Young function near infinity which makes the last integral converge. Then $A_{n}$ dominates A near infinity.

Proof. If $A(s) \equiv \infty$ for large $s$, then $A_{n} \equiv \infty$ for large $s$ also, and the conclusion is trivially true. Suppose, on the contrary, that $A$ is everywhere finite. We shall prove that, in fact, $A$ increases essentially more slowly than $A_{n}$ near infinity or, equivalently, that

$$
\lim _{r \rightarrow \infty} \bar{A}_{n}^{-1}(r) / A^{-1}(r)=0
$$

where $\bar{A}_{n}$ is the function, equivalent to $A_{n}$, defined by $\bar{A}_{n}(s)=s^{n^{\prime}}\left(\Phi_{n}\left(s^{n^{\prime}}\right)\right)^{n^{\prime}}$ for $s>0$. Observe that $\bar{A}_{n}^{-1}(r)=r^{1 / n^{\prime}} / \Omega_{n}^{-1}(r)$ for $r>0$, where $\Omega_{n}(s)=s^{n^{\prime}} \Phi_{n}(s)$ for $s>0$. Now, let $s_{0}$ be a number larger than 1 such that $\tilde{A}(s)>0$ if $s>s_{0}$. Then, since $\tilde{A}(s) / s$ increases,

$$
\begin{aligned}
\Omega_{n}(s) & \leq s^{n^{\prime}}\left(\int_{0}^{1} \frac{\tilde{A}(t)}{t^{1+n^{\prime}}} d t+\frac{\tilde{A}(s)}{s} \int_{1}^{s} t^{-n^{\prime}} d t\right) \\
& \leq s^{n^{\prime}-1} \tilde{A}(s)\left(\frac{s_{0}}{\tilde{A}\left(s_{0}\right)} \int_{0}^{1} \frac{\tilde{A}(t)}{t^{1+n^{\prime}}} d t+n-1\right) \quad \text { for } s>s_{0} .
\end{aligned}
$$


We have $s^{n^{\prime}-1} \tilde{A}(s) \leq \tilde{A}\left(s^{n^{\prime}}\right)$ if $s \geq 1$, inasmuch as $\tilde{A}$ is a Young function. Thus, if we call $c$ the last quantity in brackets in $(3.21)$, then $\Omega_{n}(s) \leq c \tilde{A}\left(s^{n^{\prime}}\right)$ for $s>$ $s_{0}$. Consequently, we deduce that numbers $r_{0}>0$ and $k>1$ exist such that $\bar{A}_{n}^{-1}(r) \leq r^{1 / n^{\prime}}\left(\widetilde{A}^{-1}(r / k)\right)^{-1 / n^{\prime}}$ for $r>r_{0}$. Therefore, thanks to $(2.5), \bar{A}_{n}^{-1}(r) \leq$ $k^{1 / n^{\prime}}\left(A^{-1}(r / k)\right)^{1 / n^{\prime}} \leq k^{1 / n^{\prime}}\left(A^{-1}(r)\right)^{1 / n^{\prime}}$ for $r>r_{0}$. Hence, (3.20) follows, since $\lim _{r \rightarrow \infty} A^{-1}(r)=\infty$.

Lemma 3. Let $A$ be a Young function and let $\alpha>-1$. Let $G$ be an open bounded subset of $\mathbb{R}^{n}, n \geq 1$, and let $G^{\prime}$ be any measurable subset of $G$ whose closure $\bar{G}^{\prime} \subset G$.

(i) If $\alpha \geq 0$, then a positive constant $C$ exists such that

$$
\|u\|_{L^{A}\left(G^{\prime}\right)} \leq C\left\|\frac{D u}{d^{\alpha}}\right\|_{L^{A}(G)}
$$

for all $u \in V_{0}^{1, A}\left(G \mid d^{-\alpha}\right)$.

(ii) If $-1<\alpha<0, \int^{\infty}(r / A(r))^{-1-1 / \alpha} d r<\infty$ and $G \in C^{0, \gamma}$ for some $\gamma \in(0,1]$, then a positive constant $C$ exists such that

$$
\|u\|_{L^{A}\left(G^{\prime}\right)} \leq C\left\|\frac{D u}{d^{\alpha \gamma}}\right\|_{L^{A}(G)}
$$

for all $u \in V_{0}^{1, A}\left(G \mid d^{-\alpha \gamma}\right)$.

Proof. Let $G^{\prime}$ be as in the statement. Then a smooth (e.g., from the class $C^{0,1}$ ) open subset $G^{\prime \prime}$ of $G$ can be chosen in such a way that $\bar{G}^{\prime} \subset G^{\prime \prime}$ and $\bar{G}^{\prime \prime} \subset G$. Clearly, we may assume, without loss of generality, that $G^{\prime \prime}$ is connected. Let $u$ be any function either from $V_{0}^{1, A}\left(G \mid d^{-\alpha}\right)$ or from $V_{0}^{1, A}\left(G \mid d^{-\alpha \gamma}\right)$, according to whether $\alpha \geq 0$ or $-1<\alpha<0$. Set $u_{G^{\prime \prime}}=\int_{G^{\prime \prime}} u(x) d x$, the mean value of $u$ over $G^{\prime \prime}$. Notice that $u_{G^{\prime \prime}}$ is well-defined, since $u \in L^{1}(G)$ by Sobolev's embedding theorem. Clearly,

$$
\|u\|_{L^{A}\left(G^{\prime}\right)} \leq\|u\|_{L^{A}\left(G^{\prime \prime}\right)} \leq\left\|u-u_{G^{\prime \prime}}\right\|_{L^{A}\left(G^{\prime \prime}\right)}+\left\|u_{G^{\prime \prime}}\right\|_{L^{A}\left(G^{\prime \prime}\right)} .
$$

We claim that a constant $c_{1}$, independent of $u$, exists such that

$$
\left\|u-u_{G^{\prime \prime}}\right\|_{L^{A}\left(G^{\prime \prime}\right)} \leq c_{1}\left\|d^{-\alpha \sigma} D u\right\|_{L^{A}(G)},
$$

where $\sigma$ is the number defined by (1.17). Indeed, we have

$$
\|D u\|_{L^{A}\left(G^{\prime \prime}\right)} \leq c_{2}\left\|d^{-\alpha \sigma} D u\right\|_{L^{A}(G)},
$$

where $c_{2}$ equals $\operatorname{diam}(G)^{\alpha}$ if $\alpha \geq 0$ and agrees with $\operatorname{dist}\left(G^{\prime \prime}, \partial G\right)^{\alpha \gamma}$ if $-1<\alpha<$ 0 . On the other hand, a constant $c_{3}$, independent of $u$, exists such that $\| u-$ $u_{G^{\prime \prime}}\left\|_{L^{A}\left(G^{\prime \prime}\right)} \leq c_{3}\right\| D u \|_{L^{A}\left(G^{\prime \prime}\right)}$. Such inequality is easily verified when $n=1$; in case $n \geq 2$, it is a consequence of Lemma 2 , which ensures that $\left\|u-u_{G^{\prime \prime}}\right\|_{L^{A}\left(G^{\prime \prime}\right)} \leq$ const. $\left\|u-u_{G^{\prime \prime}}\right\|_{L^{A_{n}\left(G^{\prime \prime}\right)}}$ (recall that $A_{n}$ is defined by (3.19)), and of Theorem 2 of $[\mathrm{C}]$, which tells us that $\left\|u-u_{G^{\prime \prime}}\right\|_{L^{A_{n}\left(G^{\prime \prime}\right)}} \leq$ const. $\|D u\|_{L^{A}\left(G^{\prime \prime}\right)}$. Thus, (3.25) holds with $c_{1}=c_{2} c_{3}$.

Now, let us consider the term $\left\|u_{G^{\prime \prime}}\right\|_{L^{A}\left(G^{\prime \prime}\right)}$. We have

$$
\left\|u_{G^{\prime \prime}}\right\|_{L^{A}\left(G^{\prime \prime}\right)} \leq \frac{\int_{G}|u(x)| d x}{m_{n}\left(G^{\prime \prime}\right) A^{-1}\left(1 / m_{n}(G)\right)},
$$

since $\|1\|_{L^{A}(G)}=1 / A^{-1}\left(1 / m_{n}(G)\right)$. On the other hand, by the classical Poincaré inequality, a constant $c_{4}$ exists such that $\int_{G}|u(x)| d x \leq c_{4} \int_{G}|D u(x)| d x$. Hence, 
by $(2.7)$,

$$
\left\|u_{G^{\prime \prime}}\right\|_{L^{A}\left(G^{\prime \prime}\right)} \leq \frac{2 c_{4}}{m_{n}\left(G^{\prime \prime}\right) A^{-1}\left(1 / m_{n}(G)\right)}\left\|d^{\alpha \sigma}\right\|_{L^{\tilde{A}}(G)}\left\|d^{-\alpha \sigma} D u\right\|_{L^{A}(G)} .
$$

Inequality (3.22) or (3.23) will follow from (3.24), (3.25) and (3.26) if we show that

$$
\left\|d^{\alpha \sigma}\right\|_{L^{\tilde{A}}(G)}<\infty
$$

Inequality (3.27) is trivially true if $\alpha \geq 0$, since $\sigma=1, d(x)^{\alpha} \leq \operatorname{diam}(G)^{\alpha}$ and $G$ is bounded. Assume now that $-1<\alpha<0, \int^{\infty}(r / A(r))^{-1-1 / \alpha} d r<\infty$ and that $G \in C^{0, \gamma}$. The convergence of the last integral ensures that $\int^{\infty} \tilde{A}(t) t^{-1+1 / \alpha} d t<\infty$ (see the Lemma of [Ta1]). Owing to (2.2) with $A$ replaced by $\tilde{A}$, the last inequality in turn implies that

$$
\int^{\infty} a^{-1}(t) t^{1 / \alpha} d t<\infty
$$

where $a$ is the derivative of $A$, so that $\tilde{A}(s)=\int_{0}^{s} a^{-1}(t) d t$. On making use of local coordinates as in the definition of the class $C^{0, \gamma}$ and recalling (3.16) one can easily show that a positive constant $c_{5}$ exists such that $m_{n}\left(\left\{x \in G: d(x)^{\alpha \gamma}>t\right\}\right) \leq c_{5} t^{1 / \alpha}$ if $t$ is sufficiently large. Hence, by (3.28),

$$
\int^{\infty} a^{-1}(t) m_{n}\left(\left\{x \in G: d(x)^{\alpha \gamma}>t\right\}\right) d t<\infty .
$$

Inasmuch as $G$ is bounded, the convergence of the last integral tells us that

$$
\int_{G} \tilde{A}\left(d(x)^{\alpha \gamma}\right) d x<\infty
$$

whence (3.27) follows. The proof is complete.

Lemma 4. Let $A$ be a Young function and let $1 \leq p<\infty$. Then

$$
\lim _{\lambda \rightarrow+\infty} \frac{\log \left(\sup _{r>0} \frac{A^{-1}(\lambda r)}{A^{-1}(r)}\right)}{\log \lambda}<\frac{1}{p}
$$

if and only if either

or

$p=1$ and a number $k>0$ exists such that

$$
s \int_{0}^{s} \frac{A(r)}{r^{2}} d r \leq A(k s) \quad \text { for } s \geq 0 .
$$

(3.31) $1<p<\infty$ and a number $k>0$ exists such that

$$
\left(\int_{k s}^{\infty}\left(\frac{r}{A(r)}\right)^{p^{\prime}-1} d r\right)^{1 / p^{\prime}}\left(\int_{0}^{s} \frac{A(r)}{r^{1+p}} d r\right)^{1 / p} \leq k \quad \text { for } s \geq 0 .
$$

The same statement is true if, in (3.29), " $\sup _{r>0}$ " is replaced by "lim $\sup _{r \rightarrow \infty}$ " and (3.30) or (3.31) holds (with 0 replaced by $\bar{s}$ as a lower limit of integration) for $s$ larger than some positive number $\bar{s}$.

Moreover, condition (3.30) holds for $s \geq 0[s \geq \bar{s}]$ if and only if $\tilde{A} \in \Delta_{2}\left[\tilde{A} \in \Delta_{2}\right.$ near infinity]. 
Sketch of the proof. The proof of the equivalence of (3.29) and (3.30) or (3.31) proceeds through the following steps.

Step 1. Inequality (3.29) holds if and only if

a number $\lambda_{0}>2$ exists such that $A^{-1}\left(\lambda_{0} r\right) \leq\left(\lambda_{0} / 2\right)^{1 / p} A^{-1}(r)$ for $r>0$.

Step 2. Condition (3.32) holds if and only if

$$
\text { a number } \mu_{0}>1 \text { exists such that } A\left(\mu_{0} s\right) \geq 2 \mu_{0}^{p} A(s) \text { for } s>0 \text {. }
$$

Step 3. If $1 \leq p<\infty$, then (3.33) is equivalent to the condition that

$$
\text { a constant } k_{1} \text { exists such that } s^{p} \int_{0}^{s} \frac{A(r)}{r^{1+p}} d r \leq A\left(k_{1} s\right) \text { for } s>0 \text {. }
$$

If $1<p<\infty$, then (3.33) is also equivalent to the condition that

a constant $k_{2}$ exists such that

$$
s^{p}\left(\int_{s}^{\infty}\left(\frac{r}{A(r)}\right)^{p^{\prime}-1} d r\right)^{1-p} \geq A\left(k_{2} s\right) \text { for } s>0 .
$$

Step 4. If $1<p<\infty$, then (3.34) and (3.35) hold if and only if (3.31) holds.

Steps 1 and 3 can be proved via arguments similar to those used in the proofs of Lemma 2 of [Ta2] and of Lemma 2.3 of [Str], respectively; step 2 is straightforward; step 4 makes use of inequalities (3.17)-(3.18). The proof of the assertion concerning the case where "sup $r>0$ " is replaced by "lim $\sup _{r \rightarrow \infty}$ " in (3.29) is similar. For the proof of the equivalence of (3.30) and of the $\Delta_{2}$ condition for $\tilde{A}$ see e.g., [BaS].

\section{One-Dimensional inequalities}

One-dimensional inequalities involving the so-called Hardy operator $H f(s)=$ $\int_{0}^{s} f(r) d r$ play a role in various contexts, for instance in the theory of interpolation, and have been extensively investigated; see [Stp] for an up-to-date survey of results. In particular, results in the framework of Orlicz space are e.g. in $[\mathrm{BK}],[\mathrm{BF}],[\mathrm{L}]$, $[\mathrm{P}]$.

Here, we deal with inequalities of the form

$$
\left\|s^{\frac{1}{1+\alpha}} \int_{0}^{s} f(r) d r\right\|_{L^{B}(0, V)} \leq C\left\|\frac{f(s)}{s^{\alpha}}\right\|_{L^{A}(0, V)}
$$

Or

$$
\left\|\frac{1}{s^{1+\alpha}} \int_{s}^{V} f(r) d r\right\|_{L^{B}(0, V)} \leq C\left\|\frac{f(s)}{s^{\alpha}}\right\|_{L^{A}(0, V)},
$$

where $V$ is either a positive number or $\infty$. The Young functions $A$ and $B$ and the real numbers $\alpha$ which make inequality (4.1) or (4.2) true for all functions $f$ such that $s^{-\alpha} f(s) \in L^{A}(0, V)$ are characterized in Theorem 5 below.

Clearly, inequalities (4.1) and (4.2) are closely related to (1.5) and (1.9), respectively. However, even though inequalities (1.1) and (1.2) are a consequence of the classical one-dimensional Hardy inequality, a derivation of (1.5) or (1.9) from onedimensional norm inequalities seems problematic when dealing with general Orlicz spaces. In fact, only the proof of necessity in Theorems 1 and 2 relies on Theorem 5 , whereas the proof of sufficiency makes use of an $n$-dimensional approach. 
Theorem 5. Let $A$ and $B$ be Young functions and let $\alpha \in \mathbb{R}$.

(i) Inequality (4.1) holds for all functions $f$ such that $s^{-\alpha} f(s) \in L^{A}(0, V)$ if and only if $\alpha>-1$ and one of the conditions (1.6)-(1.8) is fulfilled with $\bar{s}=0$ or $\bar{s}>0$, according to whether $V=\infty$ or $V<\infty$.

(ii) Inequality (4.2) holds for all functions $f$ such that $s^{-\alpha} f(s) \in L^{A}(0, V)$ if and only if $\alpha<0$ and one of the conditions (1.10)-(1.12) is fulfilled with $\bar{s}=0$ or $\bar{s}>0$, according to whether $V=\infty$ or $V<\infty$.

Remark 1. If conditions (1.6)-(1.8) or (1.10)-(1.12) are fulfilled with $\bar{s}=0$, then the constant $C$ in (4.1) or (4.2), respectively, can be shown to depend only on $\alpha$ and on $k$. If, on the contrary, $V<\infty$ and $\bar{s}>0$, then $C$ depends also on $V$ and $B$.

Remark 2. Combining Theorem 5 above with Lemma 4 of Section 3 yields necessary and sufficient conditions on $\alpha$ and $A$, of the same type as those given in Corollaries 1 and 2, ensuring that (4.1) or (4.2) holds with $A=B$. In particular, results from $[\mathrm{BF}]$ and $[\mathrm{P}]$ are recovered.

Proof of Theorem 5. Consider part i). The proof of sufficiency of conditions (1.6)(1.8) for (4.1) to hold makes use of Theorem 4 and is analogous to (and even simpler than) that of Theorem 1 . Thus, it will be omitted.

As for necessity, we shall assume that $V=\infty$, the case where $V<\infty$ being similar. Suppose that $A, B$ and $\alpha$ are such that (4.1) holds for every $f$ for which $s^{-\alpha} f(s) \in L^{A}(0, \infty)$. Hence, by Lemma 1 of [EGP] and by inequality (2.8), a positive constant $c$ exists such that

$$
\left\|r^{-1-\alpha}\right\|_{L^{B}(s, \infty)}\left\|r^{\alpha}\right\|_{L^{\tilde{A}}(0, s)} \leq c \text { for } s>0 .
$$

We claim that (4.3) can hold only if $\alpha>-1$. In fact, $\left\|r^{\alpha}\right\|_{L^{\tilde{A}}(0, s)}<\infty$ if and only if $\int_{0} \tilde{A}\left(r^{\alpha}\right) d r<\infty$. A change of variable shows that, when $\alpha<0$, the last integral converges if and only if $\int^{\infty} \tilde{A}(t) t^{-1+1 / \alpha} d t<\infty$. Hence, $\alpha>-1$, since $\tilde{A}(t) / t$ increases.

Under the assumption that $\alpha>-1$, let us now decode the information contained in (4.3). We have

$$
\begin{aligned}
\left\|r^{-1-\alpha}\right\|_{L^{B}(s, \infty)} & =\inf \left\{\lambda>0: \int_{s}^{\infty} B\left(\frac{r^{-1-\alpha}}{\lambda}\right) d r \leq 1\right\} \\
& =\inf \left\{\lambda>0: \frac{\lambda^{-1 /(1+\alpha)}}{1+\alpha} \int_{0}^{s^{-1-\alpha} / \lambda} \frac{B(t)}{t^{1+1 /(1+\alpha)}} d t \leq 1\right\} .
\end{aligned}
$$

Thus, if $F_{\alpha}$ is the Young function defined by

$$
F_{\alpha}(s)=\frac{1}{1+\alpha} s^{1 /(1+\alpha)} \int_{0}^{s} B(t) t^{-1-1 /(1+\alpha)} d t \quad \text { for } s>0,
$$

then (4.4) yields

$$
\left\|r^{-1-\alpha}\right\|_{L^{B}(s, \infty)}=\frac{s^{-1-\alpha}}{F_{\alpha}^{-1}(1 / s)} \quad \text { for } s>0
$$

In order to evaluate $\left\|r^{\alpha}\right\|_{L^{\tilde{A}}(0, s)}$, let us distinguish the cases where $\alpha>0, \alpha=0$ and $-1<\alpha<0$. If $\alpha>0$, then computations show that

$$
\left\|r^{\alpha}\right\|_{L^{\tilde{A}}(0, s)}=\frac{s^{\alpha}}{E_{\alpha}^{-1}(1 / s)} \quad \text { for } s>0
$$


where $E_{\alpha}$ is the increasing function given by $E_{\alpha}(s)=\frac{1}{\alpha} s^{-1 / \alpha} \int_{0}^{s} \tilde{A}(t) t^{-1+1 / \alpha} d t$ for $s>0$. From (4.3), (4.5) and (4.6) one obtains that $E_{\alpha}^{-1}(r) F_{\alpha}^{-1}(r) \geq r / c$ for $r>0$. Hence, since $E_{\alpha}(s) \geq\left(1-2^{-1 / \alpha}\right) \tilde{A}(s / 2)$ and $F_{\alpha}(s) \geq\left(2^{1 /(1+\alpha)}-1\right) B(s / 2)$ for $s>0$, we have $\tilde{A}^{-1}(r) B^{-1}(r) \geq r c_{1} /(4 c)$ for $r>0$, where

$$
c_{1}=\min \left\{1-2^{-1 / \alpha}, 2^{1 /(1+\alpha)}-1\right\} .
$$

Owing to (2.5), the last inequality yields $B(s) \leq A\left(c_{2} s\right)$ for $s>0$, where $c_{2}=8 c / c_{1}$. Hence, (1.6) holds with $k=c_{2}$.

Assume now that $\alpha=0$. Since $\|1\|_{L^{\tilde{A}}(0, s)}=1 / \tilde{A}^{-1}(1 / s)$, inequality (4.3) and equation (4.5) give $F_{0}^{-1}(r) \tilde{A}^{-1}(r) \geq r / c$ for $r>0$. Consequently, by $(2.5), F_{0}(s) \leq$ $A(2 c s)$ for $s>0$. Hence, (1.7) follows with $k=2 c$.

Finally, let us consider the case where $-1<\alpha<0$. Analogous arguments as above show that

$$
\left\|r^{\alpha}\right\|_{L^{\tilde{A}}(0, s)}=\frac{s^{\alpha}}{H_{\alpha}^{-1}(1 / s)} \quad \text { for } s>0
$$

where $H_{\alpha}$ is the Young function defined by $H_{\alpha}(s)=-\frac{1}{\alpha} s^{-1 / \alpha} \int_{s}^{\infty} \tilde{A}(t) t^{-1+1 / \alpha} d t$ for $s>0$. From (4.3), (4.5) and (4.7) one gets $F_{\alpha}^{-1}(r) H_{\alpha}^{-1}(r) \geq r / c$ for $r>0$. On making use of (2.5) with $F_{\alpha}$ in place of $A$, we deduce from the last inequality that $F_{\alpha}(s / 2 c) \leq \tilde{H}_{\alpha}(s)$ for $s>0$. Hence, by Lemma 5 below,

$$
\frac{1}{2 c(1+\alpha)}\left(\int_{(1+\alpha) s}^{\infty}\left(\frac{t}{A(t)}\right)^{-1-1 / \alpha} d t\right)^{-\alpha}\left(\int_{0}^{s /(2 c)} \frac{B(r)}{r^{1+1 /(1+\alpha)}} d r\right)^{1+\alpha} \leq 1 \quad \text { for } s>0 \text {. }
$$

Thus, (1.8) holds with $k=2 c(1+\alpha)$.

Part ii) follows from part i) via Fubini's theorem and inequalities (2.7) and (2.8).

Lemma 5. Let $1<p<\infty$ and let $A$ be a Young function such that

$$
\int^{\infty} \frac{\tilde{A}(t)}{t^{1+p}} d t<\infty
$$

Then

$$
\int^{\infty}\left(\frac{r}{A(r)}\right)^{p-1} d r<\infty
$$

Moreover, if $D$ is the Young function defined by

$$
D(s)=s^{p} \int_{s}^{\infty} \frac{\tilde{A}(t)}{t^{1+p}} d t
$$

then

$$
\tilde{D}(s) \leq \frac{1}{p-1}((p-1) s)^{p^{\prime}}\left(\int_{(p-1) s}^{\infty}\left(\frac{r}{A(r)}\right)^{p-1} d r\right)^{1 /(1-p)} \quad \text { for } s>0 .
$$

Proof. We begin by proving (4.9). Notice that (4.9) is trivially satisfied if $A$ equals $\infty$ for large values of the argument. Thus, we may assume that $A$ is everywhere finite. Let us set $M(r)=2 r / A^{-1}(r)$ for $r>0$. The function $M$ is increasing, since $A(s) / s$ is. Furthermore, by $(2.5), \tilde{A}^{-1}(r) \leq M(r)$ for $r>0$. Hence, by setting $M^{-1}(s)=\sup \{r: M(r)<s\}$, we obtain that $M^{-1}(s) \leq \tilde{A}(s)$ for $s>0$. Thus, (4.8) implies that $\int^{\infty} M^{-1}(t) t^{-1-p} d t<\infty$. It is easily seen, via Fubini's theorem, that the last integral converges if and only if $\int^{\infty} M(r)^{-p} d r<\infty$. A change of variable 
shows that such an inequality is in turn equivalent to $\int^{\infty}(s / A(s))^{p} a(s) d x<\infty$, where $a$ is the derivative of $A$. Thanks to (2.2), inequality (4.9) follows.

In order to prove inequality (4.11), we need to introduce some auxiliary functions which we study in separate steps. We shall assume that $A$ is strictly increasing and everywhere finite, the general case requiring minor changes.

Step 1. For every fixed $z>0$, let $\psi_{z}$ be the real-valued function on $[1, \infty)$ whose inverse is given by

$$
\psi_{z}^{-1}(\tau)=c_{z}^{1 / p}\left(\int_{\tau}^{\infty} \frac{y^{p-1}}{A(z y)^{p}} d y\right)^{-1 / p} \quad \text { for } \tau \in[1, \infty) .
$$

Here, $c_{z}=\int_{1}^{\infty} y^{p-1} A(z y)^{-p} d y$. Observe that, thanks to (4.9), the integrals on the right-hand side of (4.12) and in the definition of $c_{z}$ are convergent. It is easily seen that, for every $z>0, \psi_{z}$ is strictly increasing on $[1, \infty), \lim _{t \rightarrow+\infty} \psi_{z}(t)=+\infty$ and $\psi_{z}(1)=1$. Moreover, we claim that

$$
\begin{aligned}
\frac{1}{p-1} & \leq \int_{1}^{\infty} t^{-p} \psi_{z}(t) d t \\
& \leq \frac{1}{p-1}\left(1+c_{z}^{-1 / p^{\prime}} \int_{1}^{\infty}\left(\frac{y}{A(z y)}\right)^{p-1} d y\right) \quad \text { for } z>0 .
\end{aligned}
$$

Our claim is a consequence of the equation

$$
\int_{1}^{\infty} t^{-p} \psi_{z}(t) d t=\frac{1}{p-1}\left(1+c_{z}^{-1 / p^{\prime}} \int_{1}^{\infty}\left(\int_{\tau}^{\infty} \frac{y^{p-1}}{A(z y)^{p}} d y\right)^{1 / p^{\prime}} d \tau\right)
$$

which follows via the change of variable $t=\psi_{z}^{-1}(\tau)$ and via an integration by parts, and of the inequality

$$
\int_{1}^{\infty}\left(\int_{\tau}^{\infty} \frac{y^{p-1}}{A(z y)^{p}} d y\right)^{1 / p^{\prime}} d \tau \leq \int_{1}^{\infty}\left(\frac{y}{A(z y)}\right)^{p-1} d y
$$

a consequence of Hölder's inequality and of Fubini's theorem.

Step 2. Let us introduce the function $\omega:(0, \infty) \rightarrow(0, \infty)$ defined as $\omega(z)=$ $z \int_{1}^{\infty} t^{-p} \psi_{z}(t) d t$. By the former of inequalities (4.13),

$$
\omega(z) \geq z /(p-1) \text { for } z>0,
$$

whence $\lim _{z \rightarrow+\infty} \omega(z)=+\infty$. Furthermore, $\liminf _{z \rightarrow 0} \omega(z)=0$. Owing to (4.14), in order to prove the last assertion it suffices to show that

$$
\liminf _{z \rightarrow 0} \frac{1}{\gamma(z)} \int_{z}^{\infty} \gamma(y) d y=0
$$

where $\gamma(z)=\left(\int_{z}^{\infty} y^{p-1} A(y)^{-p} d y\right)^{1 / p^{\prime}}$. Since $A(s) / s$ increases, then $\lim _{z \rightarrow 0} \gamma(z)=$ $\infty$. Thus, (4.16) is trivially true if $\int_{0}^{\infty} \gamma(y) d y<\infty$ and easily follows via a contradiction argument when $\int_{0}^{\infty} \gamma(y) d y=\infty$.

Final step. Let $r, z>0$. By (2.4), we have

$$
r \omega(z)=\int_{1}^{\infty} r z t^{-p} \psi_{z}(t) d t \leq \int_{1}^{\infty} \frac{\tilde{A}(r t)}{t^{1+p}} d t+\int_{1}^{\infty} \frac{A\left(z \psi_{z}(t)\right)}{t^{1+p}} d t .
$$


Equation (4.12) implies that

$$
\begin{aligned}
t^{-1-p} A\left(z \psi_{z}(t)\right) & =c_{z} A\left(z \psi_{z}(t)\right)^{1-p} \psi_{z}(t)^{p-1} \frac{d}{d t} \psi_{z}(t) \\
& =c_{z}^{1 / p} t^{-p+1 / p} \psi_{z}(t)^{1 / p^{\prime}}\left(\frac{d}{d t} \psi_{z}(t)\right)^{1 / p}
\end{aligned}
$$

for $t \geq 1$ and $z>0$. Hence,

$$
\begin{aligned}
& \int_{1}^{\infty} \frac{A\left(z \psi_{z}(t)\right)}{t^{1+p}} d t \\
= & z^{p^{\prime}}\left(\int_{z}^{\infty}\left(\frac{y}{A(y)}\right)^{p-1} d y\right)^{1 /(1-p)}\left(\int_{1}^{\infty} t^{-p+1 / p} \psi_{z}(t)^{1 / p^{\prime}}\left(\frac{d}{d t} \psi_{z}(t)\right)^{1 / p} d t\right)^{p^{\prime}} .
\end{aligned}
$$

Hölder's inequality and an integration by parts give

$$
\begin{aligned}
& \left(\int_{1}^{\infty} t^{-p+1 / p} \psi_{z}(t)^{1 / p^{\prime}}\left(\frac{d}{d t} \psi_{z}(t)\right)^{1 / p} d t\right)^{p^{\prime}} \\
& \quad \leq\left(\int_{1}^{\infty} t^{-p} \psi_{z}(t) d t\right)\left(\int_{1}^{\infty} t^{1-p} \frac{d}{d t} \psi_{z}(t) d t\right)^{1 /(p-1)} \\
& \quad=\left(\int_{1}^{\infty} t^{-p} \psi_{z}(t) d t\right)\left((p-1) \int_{1}^{\infty} t^{-p} \psi_{z}(t) d t-1\right)^{1 /(p-1)} .
\end{aligned}
$$

From (4.18), (4.19), (4.15) and the definition of $\omega(z)$ we obtain

$$
\begin{aligned}
& \int_{1}^{\infty} \frac{A\left(z \psi_{z}(t)\right)}{t^{1+p}} d t \\
& \quad \leq \frac{1}{(p-1)}((p-1) \omega(z))^{p^{\prime}}\left(\int_{(p-1) \omega(z)}^{\infty}\left(\frac{y}{A(y)}\right)^{p-1} d y\right)^{1 /(1-p)} .
\end{aligned}
$$

Now, fix any $r, s>0$. Since $\omega$ is a continuous function on $(0, \infty)$ such that $\lim _{z \rightarrow+\infty} \omega(z)=+\infty$ and $\liminf _{z \rightarrow 0} \omega(z)=0$, there exists a number $z>0$ such that $\omega(z)=s$. From (4.17) and (4.20), with $z$ chosen in this way, we get that

$$
r s \leq \int_{1}^{\infty} \frac{\tilde{A}(r t)}{t^{1+p}} d t+\frac{1}{(p-1)}((p-1) s)^{p^{\prime}}\left(\int_{(p-1) s}^{\infty}\left(\frac{y}{A(y)}\right)^{p-1} d y\right)^{1 /(1-p)}
$$

Since $\int_{1}^{\infty} \frac{\tilde{A}(r t)}{t^{1+p}} d t=D(r)$, inequality (4.21) implies (4.11), by the very definition of Young conjugate.

\section{REFERENCES}

[A] R. A. Adams, Sobolev spaces, Academic Press, New York, 1975. MR 56:9247

[BaS] N. K. Bari and S. B. Stechkin, Best approximation and differential properties of two conjugate functions, Trudy Moskov Mat. Obshch. 5 (1956), 483-522. MR 18:303e

[BS] C. Bennett and R. Sharpley, Interpolation of operators, Academic Press, Boston, 1988. MR 89e: 46001

[BK] S. Bloom and R. Kerman, Weighted $L_{\Phi}$ integral inequalities for operators of Hardy type, Studia Math. 110 (1994), 35-52. MR 95f:42031

[B] D. W. Boyd, Indices for the Orlicz spaces, Pacific J. Math. 38 (1971), 315-323. MR 46:6008 
[BF] P. L. Butzer and F. Fehér, Generalized Hardy and Hardy-Littlewood inequalities in rearrangement-invariant spaces, Comment. Math. Prace Mat. Tomus Specialis in Honorum Ladislai Orlicz 1 (1978), 41-64. MR 80c:46037

[C] A. Cianchi, A sharp embedding theorem for Orlicz-Sobolev spaces, Indiana Univ. Math. J. 45 (1996), 39-65. MR 97h:46044

[EGP] D. E. Edmunds, P. Gurka and L. Pick, Compactness of Hardy-type operators in weighted Banach function spaces, Studia Math. 109 (1994), 73-90. MR 95c:47033

[H] G. H. Hardy, Note on a theorem of Hilbert, Math. Z. 6 (1920), 314-317.

[K] A. Kufner, Weighted Sobolev spaces, Teubner, Leipzig, 1980. MR 84e:46029

[L] Lai Qin Sheng, Weighted integral inequalities for the Hardy type operator and the fractional maximal operator, J. London Math. Soc. 49 (1994), 244-266. MR 95g:26029

[LT] J. Lindenstrauss and L. Tzafriri, Classical Banach spaces II, Springer-Verlag, Berlin, 1979. MR 81c: 46001

[M] B. Muckenhoupt, Hardy's inequality with weights, Studia Math. 44 (1972), 31-38. MR 47:418

[OK] B. Opic and A. Kufner, Hardy-type inequalities, Longman Scientific and Technical, Harlow, 1990. MR 92b:26028

[P] G. Palmieri, An approach to the theory of some trace spaces related to the Orlicz-Sobolev spaces (Italian), Boll. Un. Mat. Ital. 16 (1979), 100-119. MR 80f:46037

[Stp] V. D. Stepanov, Weighted norm inequalities and related topics, in Nonlinear analysis, function spaces and applications, Vol. 5, Proceedings of the spring school in Prague, Prometheus, 1994. MR 96m:26019

[Str] J. Strömberg, Bounded mean oscillation with Orlicz norms and duality of Hardy spaces, Indiana Univ. Math. J. 28 (1979), 511-544. MR 81f:42021

[RR] M. M. Rao and Z. D. Ren, Theory of Orlicz spaces, Marcel Dekker, Inc., New York, 1991. MR 92e: 46059

[Ta1] G. Talenti, An embedding theorem, in "Essays of Math. Analysis in honour of E. De Giorgi", Birkhäuser Verlag, Boston, 1989. MR 91d:46040

[Ta2] , Boundedness of minimizers, Hokkaido Math. J. 19 (1990), 259-279. MR 91g:58054

Istituto di Matematica, Facoltà di Architettura, Università di Firenze, Via dell' Agnolo 14, 50122 Firenze, Italy

E-mail address: cianchi@cesit1.unifi.it 